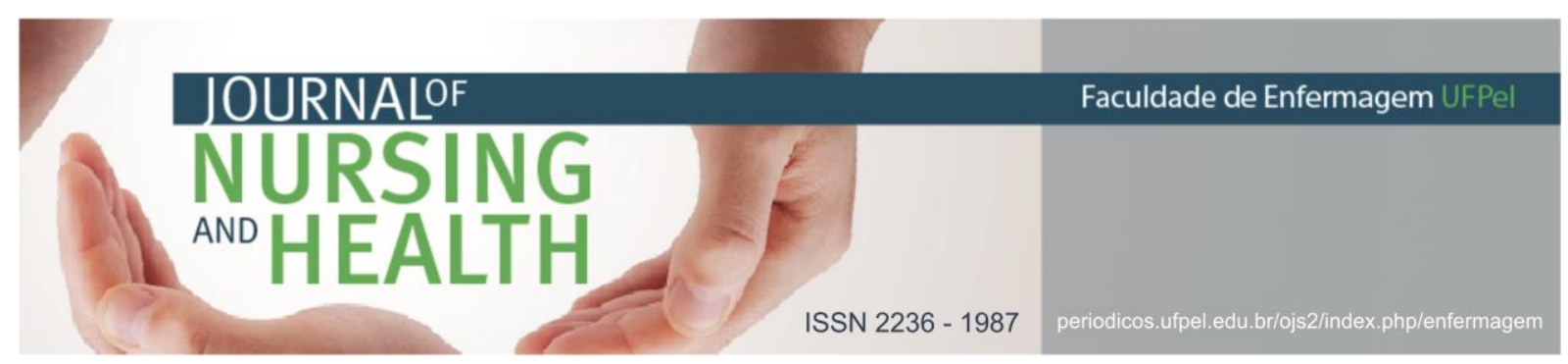

REVISÃO INTEGRATIVA

\title{
Cuidados iniciais à saúde na perspectiva cultural: produção científica sobre o tema
}

\section{Initial health care under cultural perspective: scientific production about this topic}

\section{Cuidados iniciales a la salud en la perspectiva cultural: producción científica sobre el tema}

Badke, Marcio Rossatoㅜㅜ Heisler, Elisa Vanessa²; Born, Maraísa Carine ${ }^{3}$; Almeida, Camila4 Ribeiro, Márcia ${ }^{5}$; Martorell-Poveda, Maria-Antonia ${ }^{6}$

Como citar este artigo: Badke MR, Heisler EV, Born MC, Almedia C, Ribeiro M, Martorell-Poveda MA. Cuidados iniciais à saúde na perspectiva cultural: produção científica sobre o tema. J. nurs. health. 2018;8(2):e188202

\section{RESUMO}

Objetivo: analisar os estudos existentes e disponíveis sobre cuidados iniciais à saúde, na perspectiva cultural. Métodos: revisão integrativa da literatura cujo delineamento é qualitativo e descritivo, realizada a busca em julho de 2014, nas Bases de dados e no Banco de teses da Coordenação de Aperfeiçoamento de Pessoal Nível Superior, sendo que após a aplicação dos critérios de seleção restaram 19 publicações. Resultados: o país com maior número de publicações é o Brasil, porém as pesquisas sobre a temática estão presentes nos cinco continentes, embora as produções ainda sejam pouco expressivas. A análise das produções referente aos cuidados iniciais à saúde dentro de uma perspectiva cultural, dentre os cuidados elencados estão às terapias complementares. Considerações Finais: estes achados apontam para a importância do profissional de saúde atuar considerando o contexto cultural que envolve as práticas de cuidados iniciais à saúde.

Descritores: Enfermagem; Assistência à saúde; Medicina tradicional; Conhecimento.

\section{ABSTRACT}

Objective: to analyze existing and available studies on early health care from a cultural perspective. Methods: integrative review whose delineation is qualitative and descriptive, carried out the search in July 2014, in the Databases and in the Thesis Bank of the Coordination of Improvement of Higher

1 Enfermeiro. Doutor em Ciências. Universidade Federal de Santa Maria (UFSM). E-mail: marciobadke@gmail.com http: / /orcid.org/0000-0002-9459-1715

2 Enfermeira. Mestre em Enfermagem. Universidade Federal de Santa Maria (UFSM). E-mail: elisa.vanessa@yahoo.com.br http://orcid.org/0000-0001-5438-0983

3 Enfermeira. Aluna da Residência Multiprofissional Integrada em Saúde na área de Intensivismo. Universidade Federal de Ciências da Saúde de Porto Alegre (UFCSPA). E-mail: mara.born@hotmail.com http: //orcid.org/00000003-0093-2329

4 Enfermeira. Mestre em Enfermagem. Universidade Federal de Pelotas (UFPEL). E-mail: almeidakk@yahoo.com.br http://orcid.org/0000-0003-4454-140X

5 Bióloga. Doutora em Fisiologia Vegetal. Instituto Federal Sul-Riograndense (IFSUL). E-mail: marciavribeiro@hotmail.com http: //orcid.org/0000-0002-5592-365X

6 Enfermeira. Doutora em Antropologia Médica. Universidad Rovira i Virgili, Tarragona (Espanha). E-mail: mariaantonia.martorell@urv.cat http://orcid.org/0000-0002-0309-1812 


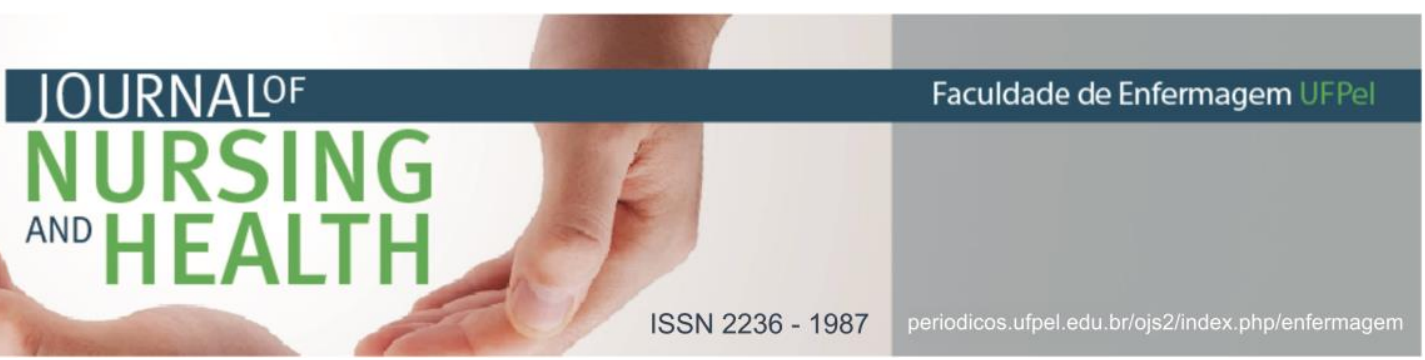

Education Personnel, and after the application of the selection criteria, there were 19 publications. Results: the country with the largest number of publications is Brazil, but research on the subject is present in five continents, although the productions are still not very expressive. The analysis of the productions related to the initial health care from a cultural perspective, among the listed care are complementary therapies. Final considerations: these findings point to the importance of the health professional to act considering the cultural context that involves the initial health care practices.

Descriptors: Nursing; Delivery of health care; Medicine traditional; Knowledge.

\section{RESUMEN}

Objetivo: analizar estudios existentes y disponibles sobre cuidados iniciales a la salud, en la perspectiva cultural. Métodos: la revisión integrativa cuyo delineamiento es cualitativo y descriptivo, realizado en la búsqueda en julio de 2014, en las Bases de datos y en el Banco de tesis de la Coordinación de Perfeccionamiento de Personal Nivel Superior y después de la aplicación de los criterios de selección quedaron 19 publicaciones. Resultados: el país con mayor número de publicaciones es Brasil, pero las investigaciones sobre la temática están presentes en los cinco continentes, aunque las producciones todavía son poco expresivas. El análisis de las producciones referentes a los cuidados iniciales a la salud dentro de una perspectiva cultural, entre los cuidados enumerados están a las terapias complementarias. Consideraciones finales: estos hallazgos apuntan a la importancia del profesional de salud actuar considerando el contexto cultural que involucra las prácticas de cuidados iniciales a la salud.

Descriptores: Enfermería; Prestación de atención de salud, Medicina tradicional; Conocimiento.

\section{INTRODUÇÃO}

Historicamente, na intenção de prover suas necessidades de sobrevivência, as sociedades acumularam saberes a partir de experimentações nos diferentes ambientes. ${ }^{1}$

Este conhecimento adquirido ao longo de suas vidas e transmitido pelas gerações mostra a existência de um cuidado que está presente ao longo da história evolutiva do ser humano durante todo o seu ciclo da vida, o nascimento, o próprio viver, o adoecer e o morrer. ${ }^{2}$ Neste discorrer, conforme pesquisa realizada no Brasil, verificase que existem diferentes maneiras de cuidar, podendo ser específico de uma determinada comunidade, tribo ou região e que também pode ser diferente do apresentado no sistema atual e oficial de saúde. ${ }^{3}$
As diferentes maneiras de cuidar, seja em qualquer etapa do ciclo da vida, podem estar atreladas à origem desta prática de saúde, ou seja, o cuidado era realizado por homens e mulheres, os quais se encontravam em épocas e contextos históricos bem distintos, influenciando assim, na maneira e na exigência de cuidado a ser prestado naquele momento e para determinada pessoa. ${ }^{3}$

Existem valores atribuídos em cada maneira de cuidar e nesta perspectiva a cultura é um aspecto importante, que deve ser levando em consideração pelos profissionais de saúde. ${ }^{4}$ Ela é composta por simbologias que são extremamente relevantes e que, se entendida por estes atores sociais, poderão ser empregadas de formas distintas na prática do cuidado, 


\section{víduo}

visando

enxergar

0

indivíduo

Neste pensar mais abrangente de cuidar está a medicina tradicional que vai além do modelo biomédico, biologicista e tecnicista, a qual consiste em uma medicina originada por crenças populares e por ações que são transmitidas a cada geração. $\mathrm{E}$, ainda inclui os ritos, as magias, a fitoterapia e outras terapias que não são explicadas pelo modelo biomédico. ${ }^{5}$

A medicina tradicional, como o próprio conceito nos mostra, foi criada para tentar enxergar e acolher a pessoa de maneira mais integral, respeitando suas particularidades, ofertando diferentes maneiras de cuidados a saúde, que podem ser desde uma simples atenção, conversa, um pegar na mão, um toque, até um cuidado mais especializado, mas sempre considerando a singularidade de cada pessoa.

Aqui cabe ressaltar o conceito de autoatenção ${ }^{6}$ que são práticas que a população utiliza tanto em nível individual como social a fim de atender os problemas subjetivos relacionados aos cuidados em saúde, sem a intervenção de profissionais cuidadores, mesmo quando estes podem ser referência para estas atividades.

Neste refletir, entendendo-se que a doença faz parte da historicidade da humanidade e que cada pessoa busca estratégias distintas para resolver seus problemas de saúde, a doença, na perspectiva social é um conhecimento de mal-estar, de que alguma coisa não vai bem, não
ISSN 2236 - 1987

essencialmente uma dificuldade biológica. O 'mal-estar' evolui para doença no momento em que se torna um objeto socialmente aceito de conhecimento e intervenção, sendo verdadeiro pelo fato de ter sua origem no senso comum. ${ }^{7}$

O tratamento de cura consiste em reconhecer além da doença manifestada, 0 cuidado requer perceber as singularidades da pessoa considerando suas crenças e seus costumes, percebendo seu contexto de interação na família e na sociedade. ${ }^{8}$ Considerando o exposto, a doença simboliza grande parte das angústias mais comuns vivenciadas pelas pessoas, famílias e sociedade, frente aos perigos e risco de adoecimento e morte acometidos durante este processo de viver.

No contexto de evitar ou prevenir que a pessoa fique doente ou que venha a falecer, surge a importância do reconhecimento das diferentes práticas populares de cuidado em saúde pelos profissionais, para que estes realizem um cuidado integral em uma perspectiva de integral. ${ }^{9}$

Para tanto, questiona-se: Como estão as pesquisas relacionadas aos cuidados iniciais à saúde dentro de uma perspectiva cultural? Assim, foi objetivo deste trabalho analisar os estudos existentes e disponíveis sobre cuidados iniciais à saúde, na perspectiva cultural.

\section{MATERIAIS E MÉTODOS}

Trata-se de uma revisão integrativa da literatura, ${ }^{10}$ a qual buscou verificar a produção acerca dos cuidados iniciais a saúde em uma 
perspectiva cultural. A pesquisa foi realizada no mês de julho de 2014, quando foi realizado um levantamento de bibliografias nas Bases de dados: Medical Literature Analysis and Retrieval System Online (MEDLINE), Scientific Electronic Library Online (SciELO), Sage Journals, Google Acadêmico e no Banco de teses da Coordenação de Aperfeiçoamento de Pessoal Nível Superior (CAPES), resultando em 295 publicações.

Os critérios adotados para a seleção das produções foram: resumos completos disponíveis online, que abordassem no título ou no resumo as palavras: "Primeiros Socorros" ou "Medicina Tradicional". A busca foi realizada nos três idiomas português, espanhol e inglês, em todas as bases consultadas. Cabe destacar, que os artigos que não contemplavam a temática foram excluídos. Com o intuito de realizar um levantamento mais abrangente das produções científicas não foi realizado um recorte temporal.

Em um segundo momento, foi realizada a leitura de todos os títulos e resumos, a exploração do material com a determinação das categorias, tratamento dos resultados e por fim, a inferência e interpretação por meio de discussão com materiais de referência na área. ${ }^{10}$

\section{RESULTADOS E DISCUSSÃO}

São apresentados os resultados de 19 referências ${ }^{11-29}$, sendo nove artigos, ${ }^{12-13,16-17,20-21,23,26,29}$ oito dissertações ${ }^{14-15,18-19,24-25,27-28}$ e duas teses. ${ }^{11,22}$
Os países que apresentaram maior número de publicações foram o Brasil com cinco publicações, ${ }^{14,18-}$ 19,24,27 a Nigéria com três, ${ }^{12-13,28}$ e o restante com uma publicação cada (África do Sul, ${ }^{15}$ Estados Unidos da América, ${ }^{17}$ Ilha do Pacífico Vanuatu, ${ }^{29}$ Itália, ${ }^{20}$ Mali, ${ }^{21}$ Nepal, ${ }^{16}$ Peru, ${ }^{22}$ Portugal, ${ }^{25}$ Tailândia, ${ }^{11}$ Tanzânia $^{26}$ e Turquia $\left.^{23}\right)$. Esse destaque para as produções brasileiras pode estar atrelado ao fato de que ocorreram incentivos a partir da década de 80 , em relação ao uso das terapias complementares e pela publicação, no ano de 2006, da Política Nacional de Práticas Integrativas e Complementares (PNPIC), que tem como objetivo ampliar as opções terapêuticas aos usuários do Sistema Único de Saúde (SUS), e consequentemente acolher seu usuário de maneira integral. ${ }^{30}$

Quanto ao ano de publicação foi constatado o inicio em 1994 com uma única publicação. ${ }^{11}$ Sendo que houve uma grande lacuna de seis anos sem publicações. Entretanto, as produções iniciaram novamente em 2001, com uma, ${ }^{12} 2002$ com três ${ }^{13-15}$ e 2003 com duas publicações ${ }^{16-17}$, em 2004 foi observada uma publicação, ${ }^{18}$ em 2005 houve outra lacuna, em 2006 uma publicação, ${ }^{19}$ em 2007 duas publicações, ${ }^{20-21}$ em 2008 uma publicação, 22009 não houve produção, 2010 com duas publicações, ${ }^{23-24} 2011$ ano sem publicações, em 2012 e 2013 duas publicações ${ }^{25-28}$ em cada ano e por fim, em 2014 uma publicação ${ }^{29}$ até o momento da pesquisa. Ante o exposto, foi observado que as publicações, vêm mantendo um rítmo constante, entretanto o número de produções 
ISSN 2236 - 1987

representa pouca exploração sobre o tema primeiros socorros.

Os continentes com maiores números de países com produções sobre a tamática foram os encontrados no continente Africano com quatro países (África do Sul, ${ }^{15}$ Nigéria, 12-13,28 Mali ${ }^{21}$ e Tanzânia $\left.{ }^{26}\right)$, seguido do continente Americano (Brasil, ${ }^{14,18-}$ 19,24,27 Estados Unidos da América ${ }^{17}$ e Peru ${ }^{22}$ ) e continente Asiático (Nepal, ${ }^{16}$ Tailândia $^{11}$ e Turquia ${ }^{23}$ ), continente Europeu (Itália ${ }^{20}$ e Portugal ${ }^{25}$ ) e a Oceania (Ilha do Pacífico Vanuatu ${ }^{29}$ ). As pesquisas sobre a temática estão presentes nos cinco continentes, o que revela 0 interesse mundial pela temática.

Dentre os materias selecionados ${ }^{11-29}$, foi constatado 0 predomínio do delineamento qualitativo em 12 pesquisas, ${ }^{11-12,14,17-}$ 19,22,24-28 seguido por quatro qualiquantitativa, ${ }^{13,15-16,29}$ uma de ensaio clínico quase experimental ${ }^{21}$ e dois trabalhos, que não informaram no seu conteúdo o tipo de pesquisa ${ }^{19,23}$.

As produções selecionadas foram analisadas, momento em que de acordo com a essência foram selecionados os materiais referentes aos cuidados iniciais à saúde dentro de uma perspectiva cultural. Esta revisão proporcionou a discussão de uma única temática que será discutida a seguir:

Cuidados iniciais a saúde no
processo de prevenção,
tratamento ou cura dos
problemas de saúde

Neste contexto, o cuidado sempre esteve presente ao longo da história evolutiva da humanidade, permeando por todo seu ciclo de vida. ${ }^{2}$ Sendo que as práticas de cuidado se constituem de conhecimento, o qual é passado de geração para geração e percebe a existência de outras maneiras de cuidar das pessoas e não só as apresentadas no sistema dito como oficial de saúde. ${ }^{3}$

Considerando o exposto, 10 dos 19 trabalhos trouxeram alguma terapia complementar no processo de prevenção, tratamento ou cura dos problemas de saúde. ${ }^{13-14,17-18,20,23,25-27,29}$

O primeiro achado é o estudo que examina o papel da água na cura entre os curandeiros tradicionais lorubás no sudoeste da Nigéria. ${ }^{12}$ Esta cura pela água é originária da antiga tradição da Yoruba, o mais comum deles são os banhos de cura, principalmente para mulher que sofrem de infertilidade. Em outro estudo, a importância da água está relacionada a sua aplicação como primeiros socorros em queimaduras e sugere a criação de um programa educacional para reduzir a morbidade e mortalidade das pessoas que sofreram esse tipo de lesão. ${ }^{24}$

A utilização do Shiatsu como instrumento complementar para redução da fadiga física dos trabalhadores de enfermagem em uma unidade hospitalar provoca mais que alívio em dores ou desconfortos físicos, ele possibilita também ao indivíduo a chance de se autoconhecer, seja nos aspectos físicos ou psicológicos. ${ }^{14}$

A perspectiva de cura de úlcera de pé para quatro vaquis nativos americanos com diabetes, apesar de métodos biomédicos e tradicionais, a cura é expressada na crença em Deus e em orações de cura. ${ }^{17}$ As práticas 


\section{JOURNALOF \\ NURSING \\ ANO HEALTH}

ISSN 2236 - 1987

tratamento de doenças pulmonares e da tuberculose dez de dezenove entrevistados disseram que iriam tratar a doença com medicamentos de curandeiros tradicionais. ${ }^{29}$

Em se tratando de práticas de autoatenção e compreendendo o cuidado à saúde e o contexto sociocultural das famílias rurais do extremo Sul do RS, as principais práticas referidas são as ações religiosas e em família, uso de plantas medicinais, dentre outras. Tais atividades são consideradas por estas famílias como os primeiros socorros realizados em casa, tendo mais de 91 citações entre os informantes. ${ }^{27}$

$\mathrm{Na}$ Tanzânia, nas áreas urbanas e rurais de sete regiões administrativas, alguns profissionais da saúde aconselham os pacientes diante de uma situação de primeiros socorros a usarem remédios, como: mel, capimlimão (Cymbopogon citratus (DC.) Stapf), espécies de babosa (Aloe sp.) entre outros. Essa indicação dos profissionais de saúde foi justificada pelo fato de que muitas pessoas utilizam continuamente a medicina tradicional/alternativa. ${ }^{26}$

Uma reflexão após a realização desta revisão revelou que não é possível generalizar os resultados, uma vez que as pesquisas devem ser direcionadas para cada localidade ou região devido às particularidades distintas e existentes em cada contexto. Entretanto, o levantamento das especificidades de cada lugar, pode contribuir com a sedimentação de maneiras de cuidar do sujeito, as quais sejam congruentes a grande maioria, entretanto, as

Corroborando ao exposto, em uma Itha do Pacífico, para o 
particularidades da minoria devem ser reconhecidas e valorizadas.

\section{CONSIDERAÇÕES FINAIS}

Esta pesquisa permitiu verificar que as publicações sobre a as práticas em saúde na perspectiva cultural tiveram seu início na década de 90 mantendo num ritmo constante, sendo que foram encontras publicações nos cinco continentes.

Os estudos mostraram a existência de terapias complementares no processo de prevenção, tratamento ou cura dos problemas de saúde, como por exemplo, o Shiatsu, a massagem, aplicação de água, entre outros, as quais muitas vezes não reconhecidas pelo sistema dito oficial de saúde.

A pesquisa constatou 0 valor atribuído ao uso das plantas medicinais como primeira opção de cuidado a saúde das pessoas, tanto no contexto individual, familiar ou social, o que sinaliza o seu emprego em diversas situações de cuidados iniciais a saúde.

Estes achados demonstram a importância e necessidade da participação dos profissionais da saúde com as práticas populares e tradicionais de cuidado, principalmente 0 enfermeiro que detém ligação direta com a população em seu contexto cultural. A participação pode se dar por meio de estudos e pesquisas envolvendo os cuidados iniciais à saúde e atuando como facilitador deste resgate de conhecimento tradicional e na sua validação científica. Assim, a qualificação profissional por meio de evidencias científica e do saber popular são fundamentais para a
ISSN 2236 - 1987

orientação destas

práticas

terapêuticas à população. A participação dos profissionais da saúde, em especial os enfermeiros, nos diálogos sobre saberes e culturas, podem proporcionar uma melhor atuação no cuidado à saúde das pessoas, contribuindo com 0 fortalecendo das diretrizes do SUS.

\section{REFERÊNCIAS}

1 Rangel $M$, Bragança FCR. Representações de gestantes sobre o uso de plantas medicinais. Rev bras plantas med [Internet]. 2009 maio[acesso em 2012 dez 10];11(1):100-9. Disponível em: http: / /www.scielo.br/pdf/rbpm/v11n 1/16.pdf

2 Freitag VL, Badke MRB. Reflexões sobre a prática do Reiki como terapia complementar no cuidado de enfermagem. In: Domingues $A$, Galhardi AC, Santánna BC, Bauer C, Rocha CF, Costa JRCC, Gomes LF, et al. Gestão em saúde: temas em debate. $1^{\text {a }}$ ed. Jundiaí-SP: Paco Editorial; 2015:200-20.

3 Badke MR, Budó MLD, Alvim NAT, Zanetti GD, Heisler EV. Saberes e práticas populares de cuidado em saúde com o uso de plantas medicinais. Texto \& contexto enferm. [Internet]. $2012 \mathrm{abr} /$ jun[acesso em $2012 \mathrm{dez}$ 10];21(2):363-70. Disponível em: http: / / www.scielo.br/scielo.php?scrip $\mathrm{t}=\mathrm{sci}$ arttext\&pid=S0104-

$07072012000200014 \& \operatorname{lng}=p t \& n r m=i s s o$

4 Geertz CA. Interpretação das culturas. $4^{a}$ ed. Rio de Janeiro: Ltc; 2015.

5 Biblioteca Virtual de Saúde (BVS). [Internet]. 2013 [acesso em $2013 \mathrm{fev}$ 


\section{NURSING \\ AND}

14].

Disponível

http: / / bvsalud.org/

6 Menéndez EL. Modelos de atenção aos padecimentos: exclusões ideológicas e articulações práticas. In: Menéndez EL. Sujeitos, saberes e estruturas: uma introdução ao enfoque relacional no estudo da saúde coletiva. $1^{a}$ ed. São Paulo: Hucitec; 2009:17-70.

7 Alves PCB, Souza IMA. Escolha e avaliação de tratamento para problemas de saúde: considerações sobre o itinerário terapêutico. In: Rabelo MCC, Alves PCB, Souza IMA. Experiência de doença e narrativa. Rio de Janeiro: Fiocruz; 1999:125-38.

8 Cruz DJL, Sousa SMA, Lima SF, Paiva SS, Gurgel WB. Cuidado cultural e doenças crônicas: análise da relação entre a teoria da diversidade e universalidade do cuidado transcultural e as necessidades da assistência de enfermagem no tratamento de doenças crônicas. Cad de pesquisa [Internet]. 2013[acesso em 2013 dez 15];20(1):43-9. Disponível em:

http: / / www.periodicoseletronicos.uf $\mathrm{ma}$.br/index.php/cadernosdepesquisa /article/view/1248/98

9 Silva RS, Matos LSL, Araújo EC, Paixão GPN, Costa LEL, Pereira A. Práticas populares em saúde: autocuidado com feridas de usuários de plantas medicinais. Rev enferm UERJ [Internet]. 2014[acesso em 2018 jun 11]; 22(3):389-95. Disponível em: http: / /www.e-

publicacoes.uerj.br/index.php/enfer magemuerj/article/view/5077

10 Botelho LLR, Cunha CCA, Macedo M. 0 método da revisão integrativa nos estudos organizacionais. Gestão e
ISSN 2236 - 1987

sociedade [Internet]. 2011[acesso em 2012 dez 10];5(11):121-36. Disponível em:

https://www.gestaoesociedade.org/g estaoesociedade/article/view/1220

11 Nuntaboot, K. Cultural perceptions of illness in rural northeastern Thailand [tese]. New Zealand (NZ): Massey University; 1994.

12 Rinne E. Water and Healing Experiences from the traditional healers inlle-Ife, Nigeria. Nordic $j$ of african studies [Internet]. 2001[cited 2013 Dec 21];10(1):41-65. Available from:

http: / / www.njas.helsinki.fi/pdffiles/vol10num1/rinne.pdf

13 Shimobiraki C, Jimba M. Traditional vs modern medicine: which healthcare option do rural Nepalese see? Technol developm [Internet]. 2002[acesso em 2013 dez 21];15(1):47-56. Disponível em:

ri.jica.go.jp/IFIC_and_JBICI-

http://jica-

Studies/english/publications/reports/ study/technology/pdf/15.pdf

14 Yamada RM. A utilização do Shiatsu como instrumento complementar para redução da fadiga física dos trabalhadores de enfermagem em uma unidade hospitalar [dissertação]. Florianópolis (SC): Universidade Federal de Santa Catarina; 2002.

15 Bereda JE. Traditional healing as a health care delivery system in a transcultural society [dissertação]. Tshwane: University of South Africa; 2002.

16 Poudyal AK, Jimba M, Murakami I, Silwal RC, Wakai S, Kuratsuji T. A traditional healers' training model in rural Nepal: strengthening their roles in community health. Trop med int 
health [Internet]. 2003 Oct[cited 2011 Jan 13];14(1):956-60. Available from: http://www.ncbi.nlm.nih.gov/pubme d/14516308

17 Vera $\mathrm{N}$ de. Perspectives on healing foot ulcers by Yaquis with diabetes. Journal of transc nurs [Internet].2003 Jan[cited 2013 Mar 20];14(1):39-47. Available from: http://www.ncbi.nlm.nih.gov/pubme d/12593269

18 Sousa IMC. Medicina alternativa nos serviços públicos de saúde: a prática da massagem na área programática 3.1 do município do Rio de Janeiro [dissertação]. Rio de Janeiro (RJ): Fundação Oswaldo Cruz; 2004.

19 Cavalcante SG. Entre a ciência e a reza: estudo de caso sobre a incorporação das rezadeiras ao programa de saúde da família no município de Maranguape CE [dissertação]. Rio de Janeiro (RJ): Universidade Federal Rural do Rio de Janeiro; 2006.

20 Leporatti ML, Impieri $M$. Ethnobotanical notes about some uses of medicinal plants in Alto Tirreno Cosentino area (Calabria, Southern Italy). J ethnobiol ethnomed [Internet]. 2007 Dec[cited 2012 Sept 15];3:34-9. Available from: http://www.ncbi.nlm.nih.gov/pmc/ar ticles/PMC2222047/

21 Willcox ML, Graz B, Falquet J, Sidibé $O$, Forster $M$, Diallo $D$. Argemone mexicana decoction for the treatment of uncomplicated falciparum malaria. Trans $r$ soc trop med hyg [Internet]. 2007 oct [cited 2013 Feb 22];101(12):1190-98. Available from: https: / /academic.oup.com/trstmh/ar ticle- abstract/101/12/1190/1904742?redire ctedFrom=fulltext

22 Gómez MEC. Acceso a los servicios de salud: Significado cultural en madres de la asociacion de shipibos artesanos en lima - ashirel [tese]. Lima: Universidad Nacional Mayor de San Marcos;2009.

23 Karaoz B, Turkey E. First-aid home treatment of burns among children and some implications at milas, turkey. J emerg nurs [Internet]. 2010 Jan[cited 2014 July 12];36(2):111-14. Available from:

http://www.ncbi.nlm.nih.gov/pubme d/?term=First-

aid+home+treatment+of+burns+among +children+and+some+implications+at+ Milas\%2C+Turkey

24 Ferraz, L. O cuidado prestado à criança pela família e comunidade indígena na percepção dos profissionais de saúde [dissertação]. Florianópolis (SC): Universidade Federal de Santa Catarina; 2010.

25 Magalhães, MJS. Anjos, magia, cabala e fé: o seu contributo para a proteção e cura das doenças e a promoção da saúde [dissertação]. Braga: Universidade do Minho; 2012.

26 Kayombo EJ, Uiso FC, Mahunnah RLA. Experience on Healthcare Utilization in Seven Administrative Regions of Tanzania. Journal of ethnobiology and ethnomedicine [Internet]. 2012 Jan[cited 2014 July 23]; (8):1-9. Available from: http://www. biomedcentral.com/cont ent/pdf/1746-4269-8-5.pdf

27 Piriz MA. Autoatenção: interfaces de cuidado por famílias rurais da região Sul [dissertação]. Pelotas (RS): Universidade Federal de Pelotas; 2013. 
28 Chimezie, RO. A Case study of primary healthcare services in Isu, Nigeria. [dissertação]. Maryland: Walden University; 2013.

29 Viney K, Johnson P, Tagaro M, Fanai $S$, Linh NN, Kelly $P$, et al. Traditional healers and the potential for collaboration with the national tuberculosis programme in Vanuatu: results from a mixed methods study. BMC public health [Internet]. 2014 Apr[cited 2014 July 10];14:(393):2-9. Available from: https://bmcpublichealth.biomedcentr al.com/track/pdf/10.1186/1471-245814-

393?site=bmcpublichealth. biomedcent ral.com

30 Ministério da Saúde (BR). Secretaria de Atenção à Saúde. Departamento de Atenção Básica. Política Nacional de Práticas Integrativas

Complementares no SUS. Brasília; 2006.

Data de submissão: $31 / 01 / 2017$

Data de aceite: 13/06/2018

Data de publicação: 28/09/2018 